\title{
The Novel Use of Endoscopy via a Cervical Oesophago-cutaneous Fistula for Removal of a Dislodged Stent in a Case of Boerhaave's syndrome
}

The Editor,

Sir,

A 41-year-old African-American male presented to the emergency room with chest and abdominal pain associated with dyspnoea following multiple episodes of emesis. Assessment and imaging revealed proximal and distal oesophageal perforations. The patient was emergently taken to the operating room where a diverting fistula of the cervical oesophagus was created through the neck.

The distal perforation was stented with a self-expandable metal stent (SEMS). The patient was discharged in stable condition with nutritional needs met by the placement of a jejunostomy tube. Follow-up at two months revealed a healed distal oesophagus but a dislodged distal stent, now present in the stomach. The gastroenterology service used the existing cervical fistula, after dilator insertion, to access the oesophagus. The dislodged stent was confirmed to be in the stomach lumen. The SEMS was retrieved with rat tooth graspers and, after manipulation using a circumferential evacuation technique, the SEMS was extracted in one piece from the dilated cervical fistula (Figs. A to D).

Boerhaave's syndrome is spontaneous perforation of the oesophagus in the absence of pre-existing pathology due to a sudden rise in intra-oesophageal pressure, typically during vomiting (1). If untreated, mortality approaches $100 \%$ and emergent surgical or endoscopic therapy with self-expandable plastic stent (SEPS) or SEMS placement is indicated $(2,3)$. Recently, encouraging results have been published utilizing stenting and diligent nonoperative care of this condition (2). This complicated case of proximal and distal perforation, utilizing an iatrogenic cervical oesophago-cutaneous fistula to remove a dislodged SEMS, has never been described in published literature to the authors' knowledge.

Boerhaave's syndrome remains a major life threatening event and the specific treatment should be selected according to each individual patient. Although classically this has been surgical, improvements in endoscopic procedures with SEMS or SEPS placement have shown decreased morbidity and mortality rates. The fistula used for endoscopy in this case was dilated prior to scope insertion to minimize further trauma to the at risk cervical oesophagus. Endoscopy may thus, be used un- conventionally, in select cases, as a viable option to surgical intervention when suitable cautionary measures are taken.

Figures A to D (from left to right below) demonstrates the use of the cervical oesophago-cutaneous fistula for endoscope insertion as well as removal of the SEMS through this same fistula employing rat-tooth graspers. The completely removed SEMS is seen in Figure D.
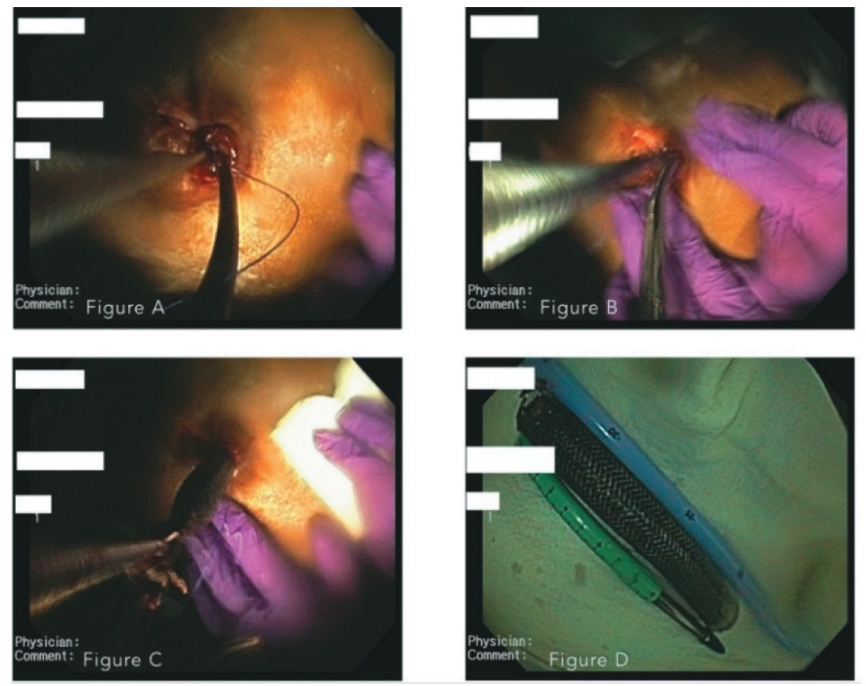

Figure: Use of cervical oesophago-cutaneous fistula.

Keywords: Boerhaave's syndrome, dislodged stent retrieval, oesophago-cutaneous fistula

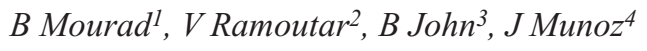

From: ${ }^{1}$ Pulmonary and Critical Care Medicine Fellow, Mount Sinai West, Icahn School of Medicine at Mount Sinai, New York, NY, USA, 10025, ${ }^{2}$ Nephrology Fellow, University of Florida, Jacksonville, FL, USA, 32209, ${ }^{3}$ Gastroenterologist, Digestive Diseases Center, San Antonio, TX, USA, 78215 and ${ }^{4}$ Gastroenterologist, Borland-Groover Clinic, Jacksonville, FL, USA, 32256.

Correspondence: Dr V Ramoutar, University of Florida College of Medicine, Jacksonville, Box L14, 653-1 W 8th Street, Jacksonville,FL, USA, 32209.email:virin.ramoutar@jax.ufl. $e d u$ 


\section{REFERENCES}

1. Blencowe NS, Strong S, Hollowood AD. Spontaneous oesophageal rupture. BMJ 2013; 346: 3095.

2. Sepesi B, Raymond DP, Peters JH. Esophageal perforation: surgical, endoscopic and medical management strategies. Curr Opin Gastroenterol 2010; 26: 379-83.
3. Eroglu A, Turkyilmaz A, Aydin Y. Yekeler E, Karaoglanoglu N. Current management of esophageal perforation: 20-years experience. Dis Esophagus 2009; 22: 374-80. 